Research and Development in Intelligent Systems XXXII, 2015, pp 213-228.

Springer, ISBN 978-3-319-25032-8, DOI 10.1007/978-3-319-25032-8_17

\title{
Development of Temporal Logic-Based Fuzzy Decision Support System for Diagnosis of Acute Rheumatic Fever/Rheumatic Heart Disease
}

\author{
Sanjib Raj Pandey ${ }^{1}$, Jixin $\mathrm{Ma}^{2}$, Chong Hong Lai ${ }^{3}$
}

\begin{abstract}
In this paper we describe our research work in developing a Clinical Decision Support System (CDSS) for the diagnosis of Acute Rheumatic Fever (ARF)/Rheumatic Heart Diseases (RHD) in Nepal. This paper expressively emphasizes the three problems which have previously not been addressed, which are: (a) ARF in Nepal has created a lot of confusion in the diagnosis and treatment, due to the lack of standard unique procedures, (b) the adoption of foreign guideline is not effective and does not meet the Nepali environment and lifestyle, (c) using (our proposed method) of hybrid methodologies (knowledge-based, temporal theory and Fuzzy logic) together to design and develop a system to diagnose of ARF case an early stage in the English and Nepali version. The three tier architecture is constructed by integrating the MS Access for backend and C\#.net for fronted to deployment of the system.
\end{abstract}

\subsection{Introduction}

This is a collaboration project between the Nepal Heart Foundation (NHF), Nepal and University of Greenwich (UoG). In this research, NHF is providing the data (patient registry), applying diagnosis procedure and practice of ARF, NHF's guidelines, providing other required expert support and information especially in ARF / RHD signs symptoms, management, medication practice etc. Moreover, NHF has continually provided the required support (aspect financial support) from an initial stage to the completion stage of research. NHF is fully participating in the evaluation of developed system and the analysis of the system's result. The training and

\footnotetext{
1 Computing \& Information System, University of Greenwich, London, SE10 9LS UK

S.R.Pandey@gre.ac.uk

2 Computing \& Information System, University of Greenwich, London, SE10 9LS UK J.Ma@gre.ac.uk

3 Mathematical Sciences Department, University of Greenwich, London, SE10 9LS UK C.H.Lai@gre.ac.uk
} 
implementation plan will be set up by NHF in conjunction with the Health Ministry of Nepal and we are giving them an appropriate training and support as required.

Based on the June 2007 - Oct 2011 [2] register of National ARF / RHD Prevention \& Control Programme from 32 Hospitals of Nepal, it was revealed that 6028 $\mathrm{ARF} / \mathrm{RHD}$ cases has been registered for secondary prevention. These patients were suffering from ARF and were receiving 3 weekly benzathine penicillin injections or oral antibiotic for secondary prevention of ARF [2].

ARF/RHD is a challenging and life threatening disease for children in the 5-15 years age groups, especially in the rural area of Nepal. In Nepal, $\mathbf{8 0 0}$ children per year die from ARF/RHD [3]. ARF/RHD can be cured at the initial stage, if we are able to make a proper diagnosis which can not only save children's lives but can also save costs and time. In Nepal, especially in rural areas, lack of awareness and most importantly ignorance of people towards the initial symptoms is a key cause of the damage. Early diagnosis of ARF/RHD will cost the patient NRs. 25.00 but the late diagnosis or negligence of the symptoms can cost them around NRs. 2, 00,000 to 3, 00,000 Lakhs [3]. In the context of the Nepalese economy, this amount is very high and the rural population cannot afford this for treatment.

According to National seminar on ARF/RHD Prevention and Control in Nepal, 2011, it has been revealed that "The lack of guideline on ARF/RHD in Nepal has created a lot of confusion in the diagnosis and treatment even among doctors. And the adoption of foreign guideline is not effective as it does not match the Nepali environment and lifestyle [3]." One of the main objective of this research is to design and develop a suitable and affordable CDSS for diagnosis of ARF at an early stage that will fit into the Nepali environment and lifestyle. Furthermore, our development diagnosis system will be a unique procedure and practice for country and individual those who are caring of ARF/RHD patients in Nepal.

The following part of this paper only focuses on the development of application. We have expressively discussed the conceptual concept and theoretical backgrounds of ARF diagnosis process in our previous paper [4].

\subsection{ARF Symptoms and Diagnosis Process}

In this section we discuss the ARF signs and symptoms, WHO criteria to diagnose of ARF and the proposed model of ARF diagnosis.

\subsection{Signs and Symptoms of ARF}

The sign and symptoms have been prepared based on the WHO, WHF and NHF's guidelines/suggestions which are given below $[5,6,7]$ 
Table 1: Sign and Symptoms of ARF

\section{Coding Sign and Symptoms}

Remarks

\begin{tabular}{cll}
\hline A. & Major Manifestations \\
$\mathbf{A r}$ & Arthritis (large joints pain or tenderness/inflamed) \\
$\mathrm{Ar}_{1}$ & Severe Pain (Ankles, Knees, Wrists, Elbows, Hips, Shoulders) \\
$\mathrm{Ar}_{2}$ & Pain Associated With Swelling, Hotness, Redness, Movement Restriction \\
$\mathrm{Ar}_{3}$ & Migratory / Shifting Severe Pain \\
$\mathbf{C r}$ & Carditis : Inflammation of the Heart Valves \\
$\mathrm{Cr}_{4}$ & Currently Present Heart Murmur, \\
$\mathrm{Cr}_{5}$ & Chest Pain / Difficulty in Breathing/ Palpitation \\
$\mathbf{C h}$ & Sydenham's Chorea : (St. Vitus Dance) \\
$\mathrm{Ch}_{6}:$ & Muscle Weakness Hands and Feet \\
$\mathrm{Ch}_{7}:$ & Twitchy and Jerking Movements of Hands or Feet or Facial Muscles \\
$\mathrm{Sn}$ & Subcutaneous Nodules \\
$\mathrm{Sn}_{8}:$ & Painless lumps on the outside surface of Wrists or Elbow or Ankles or \\
& Knees groups 3-4 (up to 12) \\
$\mathrm{Sn}_{9}:$ & Lumps round / firm and freely movable size from $0.5-2.0 \mathrm{~cm}$ \\
$\mathbf{E m}$ & Erythema Marginatum \\
$\mathrm{Em}_{10}:$ & Painless, flat pink patches on the skin \\
$\mathrm{Em}_{11}:$ & Not itchy or painful and has well-defined borders \\
$\mathbf{B}$. & Minor Manifestations \\
$\mathrm{Fe}_{12}$ & Fever \\
$\mathrm{Art}_{13}$ & Arthralgia (joints pain) \\
$\mathrm{Ecg}_{14}$ & Prolonged P-R interval on ECG \\
$\mathrm{Crp}_{15}$ & Raised or Positive CRP \\
$\mathrm{Esr}_{16}$ & Raised ESR \\
$\mathbf{C .}$ & Mandatory/Essential \\
$\mathrm{Prt}_{17}$ & Positive Rapid Strep Test \\
$\mathrm{Ast}_{18}$ & Raised (Positive) Anti-Streptolysin O tire (ASOT) \\
$\mathrm{Gs}_{19}$ & Positive throat culture for GAS infection \\
\hline
\end{tabular}

\subsection{Diagnosis Process of ARF}

According to the WHO guidelines $[6,8]$ the diagnosis process of ARF are as follows:

- The First episode of ARF can be confirmed if

o There is at least one observable MAJOR symptom and at least two MINOR symptoms manifested plus evidence of GAS infection.

- Recurrent ARF (with no RHD) can be confirmed if 
- There is at least one observable MAJOR symptom and at least two MINOR symptoms manifested plus evidence of GAS infection.

- Recurrent ARF (with existing RHD) can be confirmed if

Two MINOR symptoms plus evidence of GAS infection

\subsection{Purposed model for diagnosis of ARF}

The purposed model for the diagnosis of ARF in Nepal is given below. This diagnosis model has been prepared based on the expertise guidelines and met the Nepali life style and environment.

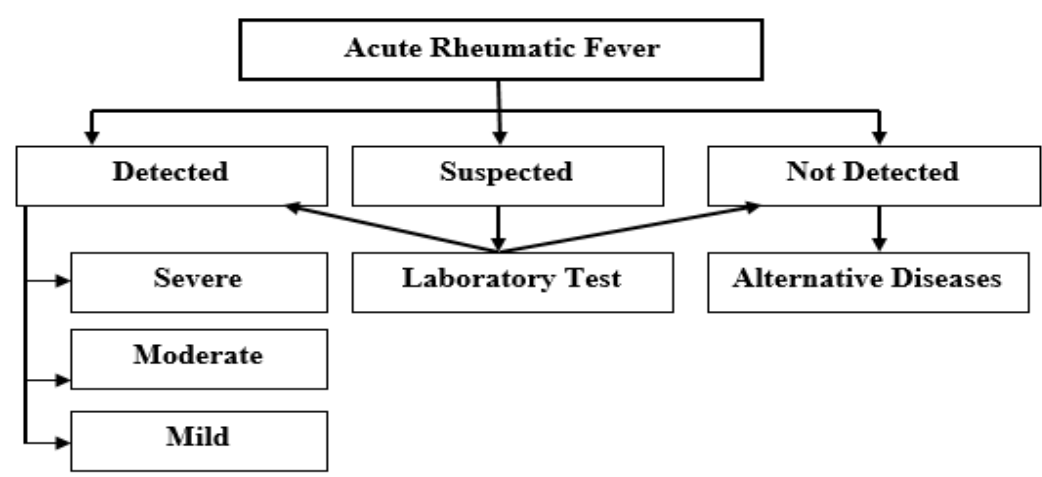

Fig. 1 Purposed model for diagnosis of ARF.

\subsection{Signs and symptoms for different level of the severity}

Based on the NHF's expertise's guidelines, we have separated the signs and symptoms for different level of the severity that are presented in the below. Based on these signs and symptoms we identify the severity level of ARF for patients. 


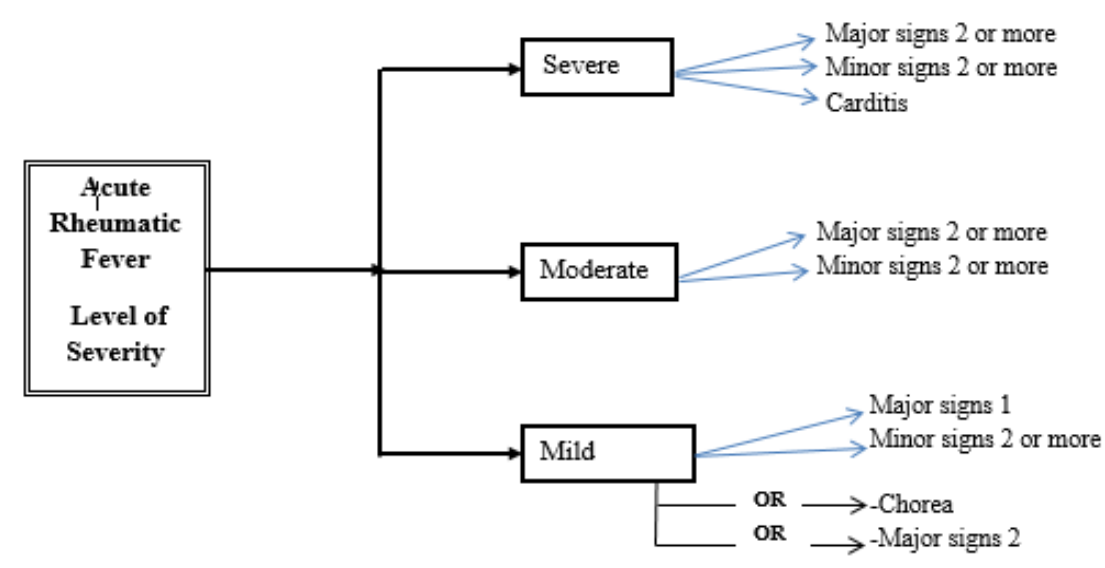

Fig. 2. Signs and symptoms for different level of the severity.

\subsection{Methodology}

In this section we discuss the applied hybrid methodology's structure and its components. Proposed hybrid methodology is a combination of knowledge-based, temporal and fuzzy logic. It is a three model structure that is integrated into one umbrella and applied for designing and deploying the ARF diagnosis model. The structure of hybrid methodology is given below: 


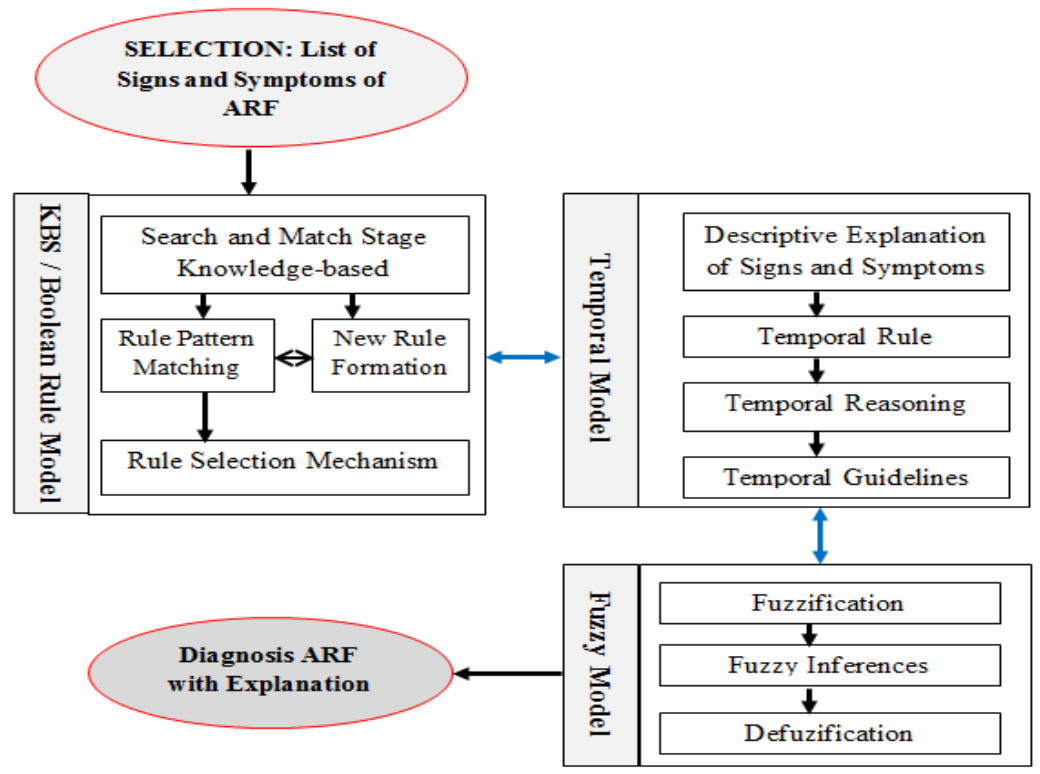

Fig. 3 The structure of hybrid methodology.

\subsection{Knowledge-base and rules to diagnose of ARF}

A knowledge-based model is responsible to identify the ARF status (Detected, Suspected and Not Detected) and the severity level of ARF and construction of the rules. The ARF status and the severity level of ARF has been identified by the presented patients' signs and symptoms. Depending upon the observed traits in a detected ARF patient, the system can diagnose the level of the severity of ARF into one of four categories: suspected, severe, mild or moderate. This categorization (level of the severity) and associated symptoms is shown in figure 2. The following equations (1-7) have been created to identify the level of the severity of ARF.

Severe $=\{$ carditis $\wedge$ essential $\geq 1 \wedge$ major $\geq 2 \wedge$ minor $\geq 2\}$ .1

Moderate $=\{$ essential $\geq 1 \wedge$ major $\geq 2 \wedge$ minor $\geq 2\} \ldots \ldots \ldots \ldots \ldots \ldots \ldots . . . \ldots$

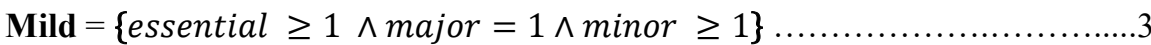

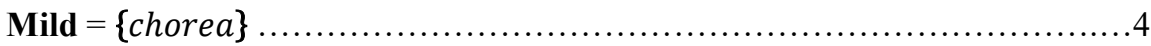

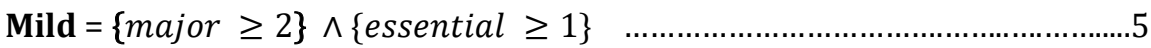


Mild $=\left\{\operatorname{Art}_{13} \wedge \mathrm{Fe}_{12} \wedge \mathrm{Crp}_{15} \wedge\right.$ essential $\left.\geq 1\right\}$

Suspected $=\{$ major $=1 \wedge$ minor $=1 \wedge$ essential $\geq 1\} \ldots \ldots \ldots \ldots \ldots \ldots \ldots \ldots \ldots$

Suspected $=\left\{\mathrm{Art}_{13} \wedge \mathrm{Fe}_{12} \wedge\right.$ essential $\left.\geq 1\right\} \ldots \ldots \ldots \ldots \ldots \ldots \ldots \ldots \ldots \ldots \ldots . . . \ldots$

We are capturing the patients' sign and symptoms in Boolean expression for example "True" or "False", "0" or "1" etc. We are using "P=present" if signs are presented or observed and " $\mathrm{A}=\mathrm{absent}$ " if signs are not presented or not observed. The reason for applying Boolean expression is that it can easily capture signs and symptoms for doctors and rural health workers as well as being easy to apply in rule formation. Furthermore, "Boolean principles also underlie logical checking of rulebased decision support systems for inconsistencies, incompleteness and redundancy" [10]. A set of rules have been created for all the severity level of ARF. Each rule has symptoms in the condition part and corresponding severity level of ARF with numbers of positive symptoms are consisting in consequence part.

We have applied the mixed reasoning methods (both backward and forward reasoning/chaining). In the system some of the rules are specially designed and used for forward reasoning and others are used for backward reasoning. The purpose of applying the mixed reasoning process is to maximize the diagnosis efficiency of ARF.

\subsubsection{Search and Match Stage (SMS)}

SMS is designed and developed to identify the ARF status and the severity level of ARF, based on the observed patients' signs and symptoms and the expertise guidelines. SMS process has 3 different sub components, they are; Rule Pattern Matching (RPM), New Rule Formation (NRF) and Rule Selection Mechanism (RSM). Each component is a sub process of SMS and implemented by a set of algorithms that are explained below. The steps of the algorithm is given below:

\footnotetext{
Step 1: START $\rightarrow$ Read and capture all signs and symptoms of patients

Step 2: Define array and store all selected signs and symptoms

Step 3: Create three memory variables to keep the number of major, minor and essential, signs and symptoms from array (which is define in Step 2).

Step 4: Read all signs and symptoms from array

Step 5: Have all signs and symptoms are read from array?

Step 6: IF NO- checks and matches the signs with KBS (ARF manifestation) and identify the satisfied sign then increment value by 1 in appropriate memory variable for example major $=$ major +1 or minor $=$ minor +1 or essential $=$ essential +1 .

Step 7: Go to next symptoms read (Step 4) and in the Step 5, IF YES then

Step 8: Read the value from three variables (major, minor \& essential) and follow the guidelines of KBS, calculate observed sings symptoms and identify the severity level of ARF Step 9: Display the severity level of ARF with justifications and END
} 


\subsubsection{Rule Pattern Matching (RPM)}

Pattern matching is the process of matching the new or existing facts against the rule or search for a rule that matches the pattern of data. There are various algorithms used for pattern matching Linear, Rete, Treat, Leaps etc. Here, we used very simplistic pattern matching strategy. The pattern matching task is performed by the inference engine. Pattern matching process will help to fire the exact matching rule by eliminating the unsuitable rules and facts.

After identifying the ARF status, the RPM will automatically activate and identify the exact rule that matches between the pattern and fact (observed signs and symptoms). The pattern of the rule matches the facts in working memory; then the activation of the rule will be fired.

\subsubsection{New Rule Formation (NRF)}

During the process of RPM, If rule has not been found in the rule-based then NRF is activated which has "Auto Rule Generate Algorithm (ARGA)" that generates a new rule. ARGA is a self-developing algorithm that is applied in this system to formation of new rule automatically. The algorithm is created based on the signs and symptoms for different severity level of ARF and expertise guidelines. This algorithm is very suitable for this system to generate a new rule. However, expertise can revised, delete or add new parameters in the added rule, if required. The benefit of using this algorithm is, to add the new rule, require the domain expertise, knowledge engineer and programmer. So it is time consuming process (depended upon availability of all expertise). Therefore, NRF process act all task (like expertise, knowledge-engineer and programmer) automatically and add the new rule on the rule-based system. The steps of ARGA algorithm is describe below:

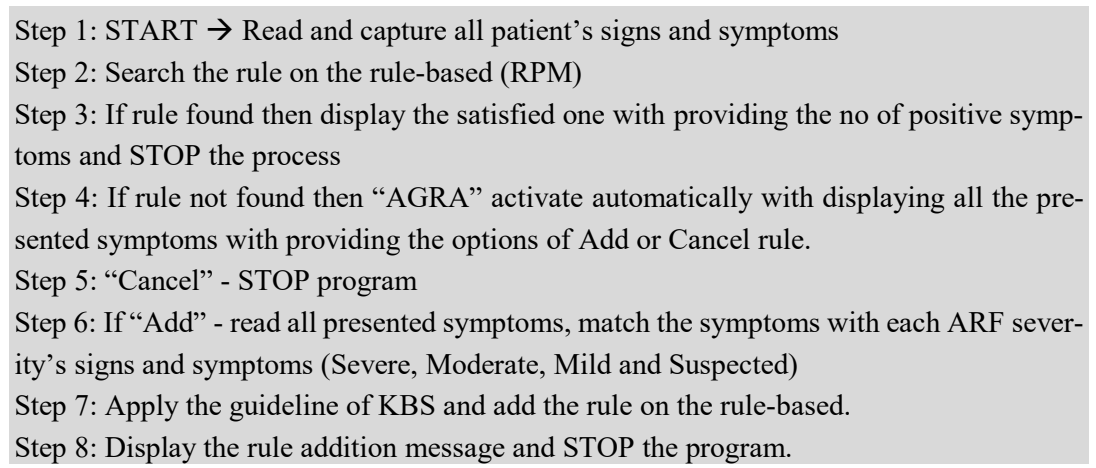

\subsubsection{Rule Selection Mechanism (RSM)}

In this system, RSM concept is simple but very important to the diagnosis of ARF. During the RPM process, it will show only matching rule and based on that 
make a decision, which will not be accurate enough for the system. Therefore, the main reason to develop and implement this RSM is to diagnose of ARF more effectively. RSM is responsible to search for and select the appropriate rules from every level of the severity - severe, moderate, mild and suspected based on the given total number of positive signs.

\subsection{Temporal Logic \& Temporal Rule (TR)}

The temporal dimension for each signs and symptoms development time is divided up to 24 weeks. Temporal knowledge-based is designed as a temporal lookup table [4]. The sign "GAS infection on the throat" will be a starting time point or origin of time for individual patient. Other captured signs and symptoms presented or development time will be calculated from origin of time and we find out where the particular symptoms consist based on the temporal lookup table. The following equation is used to show the temporal dimension and create a temporal knowledgebased.

Table 2. The time span of ARF's signs and symptoms (in days)

\begin{tabular}{|c|c|c|c|c|c|}
\hline $\begin{array}{l}\text { Signs and } \\
\text { Symptom }\end{array}$ & Time Period & $\begin{array}{l}\text { Absolutely posi- } \\
\text { tive }\end{array}$ & Very Positive & $\begin{array}{l}\text { Relatively } \\
\text { Positive }\end{array}$ & Suspected \\
\hline Arthritis & $\begin{array}{l}\left(\mathrm{P}_{11}, \mathrm{P}_{12}\right] \\
\mathrm{x}=\mathrm{P}_{12}-\mathrm{P}_{11}\end{array}$ & $x>14 \wedge x<=35$ & $\begin{array}{l}(x>=0 \wedge x<=14) \vee \\
(x>35 \wedge x<=49)\end{array}$ & $x>49 \wedge x<=63$ & $\begin{array}{l}x>63 \wedge \\
x<=168\end{array}$ \\
\hline Carditis & $\begin{array}{l}\left(\mathrm{P}_{21}, \mathrm{P}_{22}\right] \\
\mathrm{x}=\mathrm{P}_{22}-\mathrm{P}_{21}\end{array}$ & $x>21 \wedge x<=42$ & $\begin{array}{l}(x>=0 \wedge x<=21) \vee \\
(x>42 \wedge x<=56)\end{array}$ & $x>56 \wedge x<=77$ & $\begin{array}{l}x>77 \wedge \\
x<=168\end{array}$ \\
\hline EM & $\begin{array}{l}\left(\mathrm{P}_{31}, \mathrm{P}_{32}\right] \\
\mathrm{x}=\mathrm{P}_{32}-\mathrm{P}_{31}\end{array}$ & $x>14 \wedge x<=35$ & $\begin{array}{l}(x>=0 \wedge x<=14) \vee \\
(x>35 \wedge x<=140)\end{array}$ & $\begin{array}{l}x>140 \\
\wedge x<=168\end{array}$ & $x>168$ \\
\hline Chorea & $\begin{array}{l}\left(\mathrm{P}_{41}, \mathrm{P}_{42}\right] \\
\mathrm{x}=\mathrm{P}_{42}-\mathrm{P}_{41}\end{array}$ & $x>35 \wedge x<=98$ & $\begin{array}{l}(x>21 \wedge x<=35) \vee \\
(x>98 \wedge x<=168)\end{array}$ & $x>7 \wedge x<=21$ & $\begin{array}{r}x>=0 \wedge \\
x<=7\end{array}$ \\
\hline \multicolumn{2}{|c|}{ Subcutaneous $\left(\mathrm{P}_{51}, \mathrm{P}_{52}\right]$} & $x>49 \wedge x<=98$ & $(x>35 \wedge x<=49) \vee$ & $(x>21 \wedge$ & $(\mathrm{x}>=0 \wedge$ \\
\hline Nodules & $\mathrm{x}=\mathrm{P}_{52}-\mathrm{P}_{51}$ & & $(x>98 \wedge x<=119)$ & $\begin{array}{c}\mathrm{x}<=35) \vee \\
(\mathrm{x}>119 \wedge \\
\mathrm{x}<=147)\end{array}$ & $\begin{array}{r}x<=21) \vee \\
(x>147 \wedge \\
x<=168)\end{array}$ \\
\hline $\begin{array}{l}\text { Minor Symp- } \\
\text { toms }\end{array}$ & $\begin{array}{l}\left(\mathrm{P}_{61}, \mathrm{P}_{62}\right] \\
\mathrm{x}=\mathrm{P}_{62}-\mathrm{P}_{61}\end{array}$ & $x>7 \wedge x<=49$ & $\begin{array}{l}(x>=0 \wedge x<=7) \vee \\
(x>49 \wedge x<=70)\end{array}$ & $x>70 \wedge x<=91$ & $\begin{array}{r}X>91 \wedge \\
x<=168\end{array}$ \\
\hline
\end{tabular}

The sample equation of temporal relation between ARF's signs for absolutely positive are given below:

\subsubsection{Relation between arthritis \& other symptoms: Absolutely Positive Case}


- Arthritis and Carditis

$\operatorname{Occurs}\left(\operatorname{arthritis}, p_{11}, p_{12}\right) \wedge \operatorname{Occurs}\left(\operatorname{carditis}, p_{21}, p_{22}\right) \wedge p_{21}-p_{11}=7 \wedge$

$p_{22}-p_{12}=7 \wedge p_{12}-p_{21}=14 \wedge p_{22}-p_{11}=28$,

where $\left\{\begin{array}{l}14<p_{12}-p_{11} \leq 35 \\ 21<p_{22}-p_{21} \leq 42\end{array}\right.$

- Arthritis and Erythema Marginatum (em)

$\operatorname{Occurs}\left(\operatorname{arthritis}, p_{11}, p_{12}\right) \wedge \operatorname{Occurs}\left(e m, p_{31}, p_{32}\right) \wedge p_{11}=p_{31} \wedge p_{12}=p_{32} \wedge$

$p_{12}-p_{31}=21 \wedge p_{32}-p_{11}=21$, where $\left\{\begin{array}{l}14<p_{12}-p_{11} \leq 35 \\ 14<p_{32}-p_{31} \leq 35\end{array}\right.$

- Arthritis and Chorea

$\operatorname{Occurs}\left(\operatorname{arthritis}, p_{11}, p_{12}\right) \wedge \operatorname{Occours}\left(\operatorname{chorea}, p_{41}, p_{42}\right) \wedge p_{41}-p_{11}=21 \wedge$

$p_{12}=p_{41} \wedge p_{42}-p_{12}=63$, where $\left\{\begin{array}{l}14<p_{12}-p_{11} \leq 35 \\ 35<p_{42}-p_{41} \leq 98\end{array}\right.$

- Arthritis and Subcutaneous Nodules (sn)

$\operatorname{Occurs}\left(\operatorname{arthritis}, p_{11}, p_{12}\right) \wedge \operatorname{Occours}\left(\operatorname{sn}, p_{51}, p_{52}\right) \wedge p_{51}-p_{12}=14 \wedge p_{51}-$

$p_{11}=35 \wedge p_{52}-p_{12}=63$, where $\left\{\begin{array}{l}14<p_{12}-p_{11} \leq 35 \\ 49<p_{52}-p_{51} \leq 98\end{array}\right.$

- Arthritis and Minor Symptoms (ms)

$\operatorname{Occurs}\left(\operatorname{arthritis}, p_{11}, p_{12}\right) \wedge \operatorname{Occurs}\left(m s, p_{41}, p_{42}\right) \wedge p_{11}-p_{61}=7 \wedge p_{62}-$ $p_{12}=14 \wedge p_{12}-p_{61}=28$, where $\left\{\begin{array}{l}14<p_{12}-p_{11} \leq 35 \\ 7<p_{62}-p_{61} \leq 49\end{array}\right.$

\subsubsection{Temporal Reasoning (TR) and Temporal Guideline (TG)}

TR is to determine and analyse the relation between symptoms as well as provide the linguistic information how closely related a particular symptom with ARF. Temporal abstraction will be applied to analyse the relation between the symptoms and absolute-time granularity, will capture the temporal dimension of symptoms and its relationship with ARF is analysed. We created point-based time patterns of symptoms and, based on these time dimension, we introduce how precisely related of particular signs with ARF $[4,13,14]$.

The guideline information is designed in linguistic variable for instances "Absolutely Positive for ARF", "Very Positive for ARF", "Relatively Positive for ARF" and "Suspected for ARF". This linguistic base information will provide support for rural health workers to understand the relation between symptoms and ARF. The linguistic variable and time frame is designed based on the expertise support from Nepal. Temporal rule and temporal reasoning will interpret each patient's symptoms and how to precisely relate with ARF. 


\subsection{Fuzzy Logic}

The main concept of fuzzy logic is that a statement cannot be restricted to being only true or false; it must have some freedom of partial truth. Moreover, Fuzzy logic has a great potential to use linguistic variables, for example few, slow, fast, large, heavy, severe, mild, low, medium, high, short, average, tall etc. [11] Monotonically Increasing Linear Membership Function (MILMF), Root Sum Square, and Centre of Gravity methods are applied for fuzzification, fuzzy inference and defuziffication process respectively. MILMF converted the crisp value into fuzzy value for this system. The monotonically increasing linear membership function and equation is given below [12].

$$
L(x ; \alpha, \beta)= \begin{cases}0 & x<\alpha \\ \frac{x-\alpha}{\beta-\alpha} & \alpha \leq x \leq \beta \\ 1 & x>\beta\end{cases}
$$

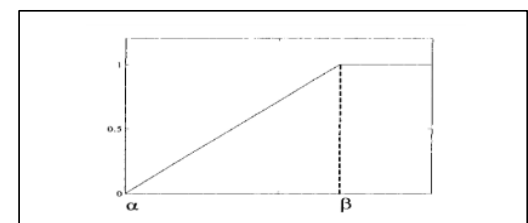

Table 3. Input variables, range and fuzzy set.

\begin{tabular}{lll}
\hline Input Variables & Ranges & Fuzzy Sets \\
\hline All ARF signs and symptoms that presented in & 0 & None \\
above table 3. & $0-1$ & Fairly Positive \\
& $1-2$ & Moderately Positive \\
& $2-3$ & Absolutely Positive \\
\hline
\end{tabular}

\subsubsection{Fuzzy Inferences}

MILMF is applied to determine the degree of symptoms to ascertain the doctor's belief of the symptoms' severity. The fuzzy inference mechanism will map the entire set of rules with membership degrees. Then the output of each rule is aggregated and Mamdani's Centre of Gravity (CoG) method is applied for the defuzzification process. Root Sum Square (RSS) will apply for the getting the values from firing rules. To achieve this following equation is applied.

$R v_{i} \ldots \ldots v_{n}=$ Value of firing Rules

Ovi.......Ov $v_{n}=$ Output value of RSS

$\mathrm{Ov}_{\mathrm{i}}=\sqrt{R v_{i}^{2}}$

The equation of defuzzification process given below:

$\operatorname{COG}(\mathrm{Y})=\frac{\sum \mu_{y}\left(x_{i}\right) x_{i}}{\sum \mu_{y}\left(x_{i}\right)}$

Where,

$\mu_{y}\left(x_{i}\right)=$ Membership value in the membership function

$\mathrm{x}_{\mathrm{i}}=$ centre value of membership function 


\subsection{System Development}

This application is developed by applying three-tier concept. The three-tier architecture enables to increase performance, easy to maintain code, flexibility and

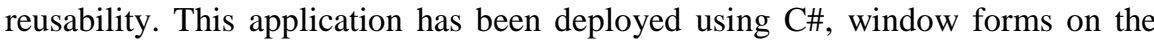
front-end and MS access as back-end. The front-end is developed to be very simple and easy to use and consists of various forms for example login, patient registration, check-up, patient history, help/guidelines etc. The development environment of system is Visual Studio.Net 2012 and Framework is .Net Framework 4.0. The user interface is Windows and the programming language is C\#.net. MS Access 2010 is used for database and ADO.NET is using for data access. The components of layered architecture are given below:

Presentation layer / Graphical User Interface (GUI): Presentation layer contains the user screen of various programs or forms associated with GUI components and code-behind it which handles the assign instruction appropriately. This layer is delivers the information to the user and to the system.

Data Layer: This layer has other sub components; the business service methods, data access logic, data model and all required SQL coding. Business service is a method to communication with the data access. This service is calling the data access methods and delivering the result presentation layer. Data model is a set of entities (table) where we stored the records. SQL code is a set of statements that is used to manipulate the data.

Data Access: Data access task is to accessing the data on the database via SQL code.

\subsection{User Interface for diagnosis of ARF Application}

This application has various input and output forms. User logon, patient's registration, view patients history/report, diagnosis, patient report, view/update rules etc. Only registered user can use the application. Expertise can update, modify, delete, and add rules etc. Moreover, expertise is responsible to manage all the rules and help/guideline information. The sample interfaces of the application are given below: 
Development of Temporal Logic - Based Fuzzy DSS for Diagnosis of Acute Rheumatic Fever 13

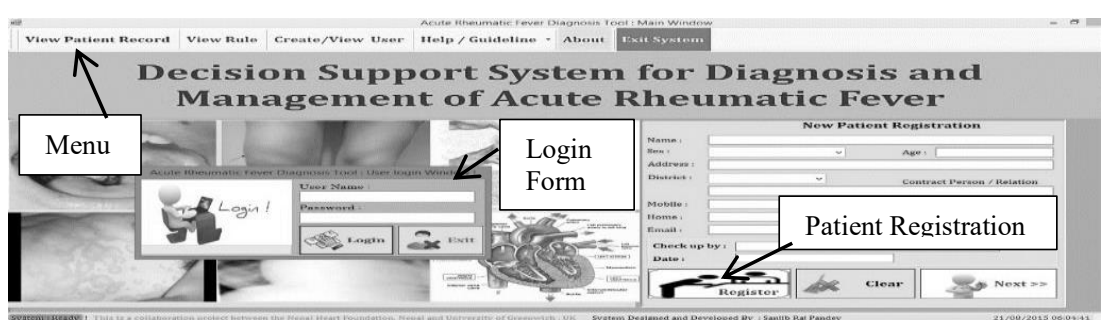

Fig.4 User login, application's main window and new patient registration.

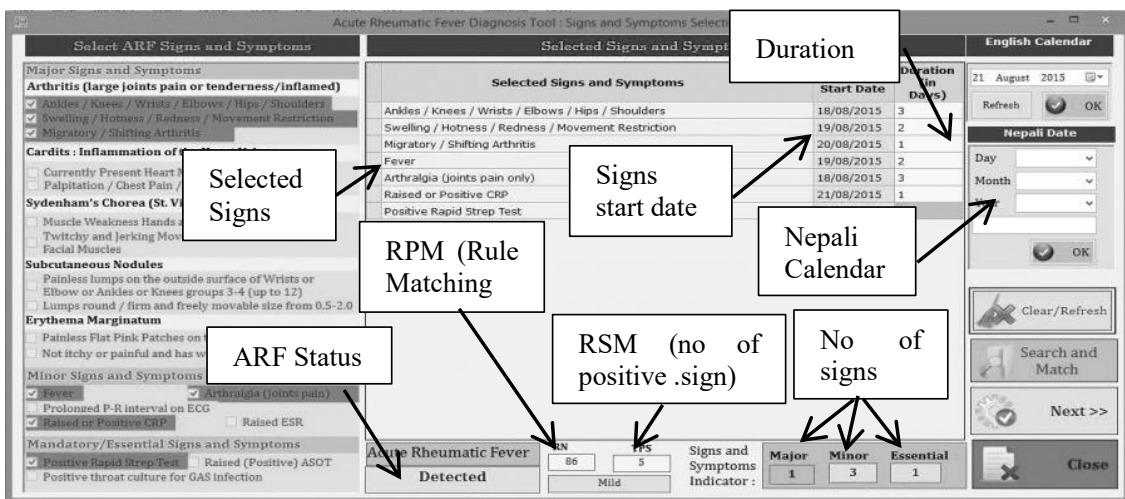

Fig. 5 Patient's signs \& symptoms selection form.

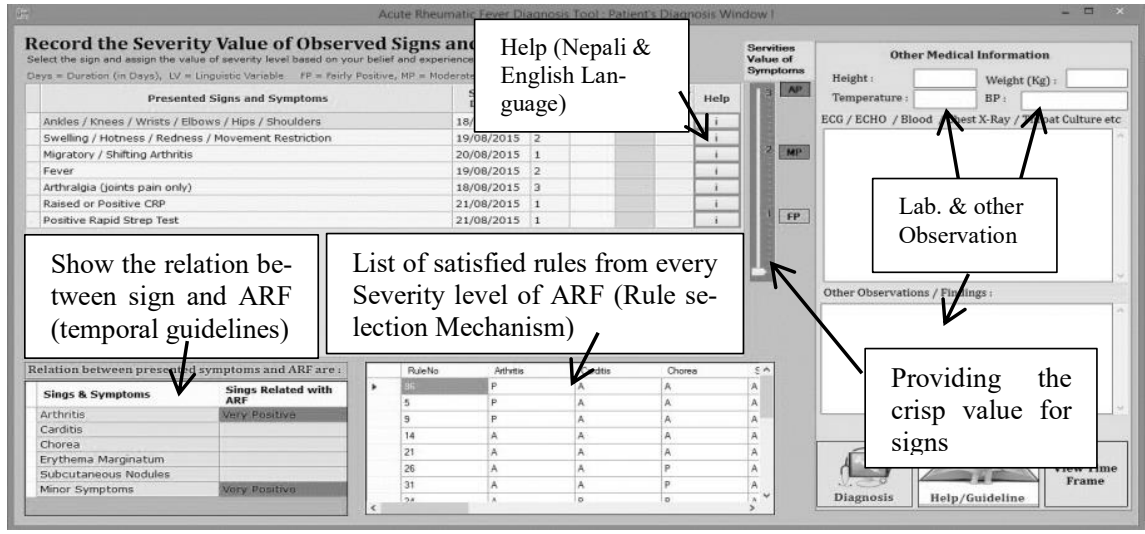




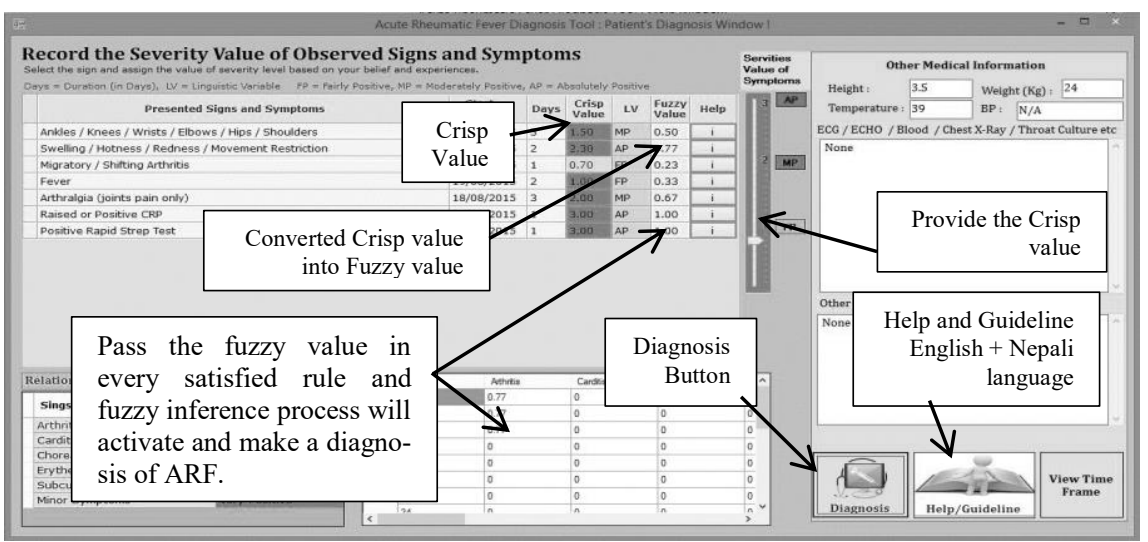

Fig. 6 Recording the fuzzy value \& other (e.g. laboratory result, other observations).

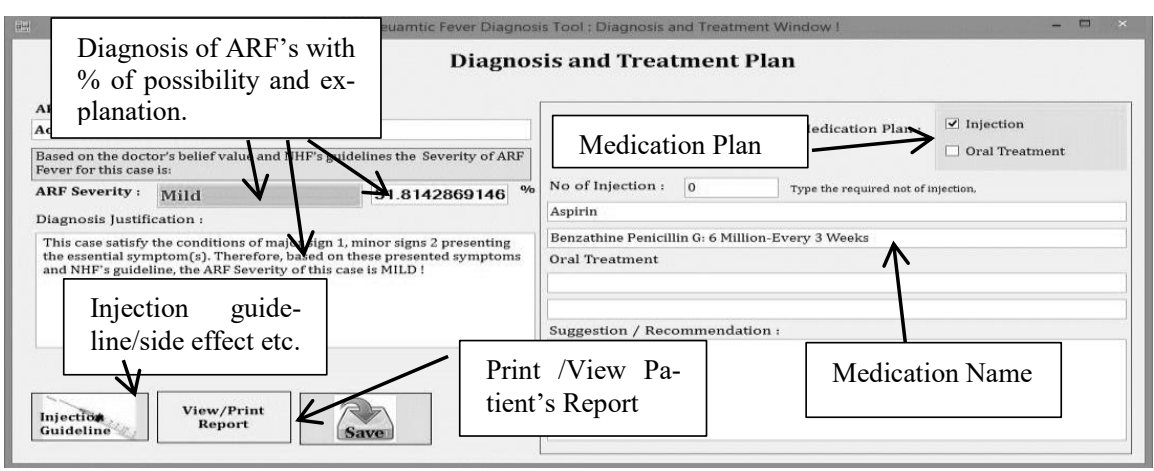

Fig. 7 Diagnosis of ARF and further information.

\subsection{System Testing and Conclusion}

The developed system has been tested with NHF's patients' registry data (585 records) sets and it reflects that $93.6 \%$ were matches. Therefore, we proved that this hybrid methodology can handle both temporal abstraction and uncertainty in an integrated way and shown to be effective in the diagnosis of ARF at an early stage.

Knowledge-based model captured the required information for the diagnosis of ARF. Then we presented ARF diagnosis model that is divided into four severity level of ARF based on the expertise guidelines from Nepal. We developed a rule for the diagnosis of ARF in different level of the severity according to KBS. In the second stage we applied temporal theory to show the relation between the symptoms and analysed them. Then we analysed the ARF's symptoms development time frame and created a temporal rule by explaining the relation of the each sign with ARF in linguistic strength. These are: absolutely positive, very positive, relatively positive and suspected. The temporal reasoning process explained the linguistic 
strength of signs and symptoms and how precisely related they are with ARF. Then we applied fuzzy logic to handle the uncertainty as well as make a decision of ARF based on the qualitative precise temporal guidelines.

Our hybrid methodologies proved to be an appropriate solution to the development of a decision support system for ARF. It is very useful to diagnose ARF an early stage where manpower and technologies are not guaranteed. It is cost effective, easy to use, flexible, and suitable for the Nepali environment and lifestyle and introduces the unique procedure for the diagnosis of ARF in Nepal.

References

[1] Shahid Gangalal National Health Centre, Nepal “Annual Report", 2007 http://www.sgnhc.org.np/report-2007/main\%20Page\%201\%20to\%2066.pdf accessed 27 Feb. 2015

[2] Regmi PR, et al, "Prevalence of Sydenham's Chorea in Patients with Acute Rheumatic Fever in Nepal" www.nepjol.info/index.php/nhj/article/download/8345/6794 accessed 3 April 2015.

[3] National Seminar on RF/RHD Prevention and Control in Nepal, July, 2011

[4] Sanjib Pandey, Jixin Ma, Choi-Hong Lai, Prakash Raj Regmi “A Conceptual Framework to Diagnosis of Acute Rheumatic Fever Based on the Temporal and Fuzzy Logic Approach" Candian International Journal of Science and Technology, ISBN 8472356X (accepted)

[5] "Rheumatic Fever and Rheumatic Heart Disease", Report of a WHO Expert Consultations, Geneva, 29 Oct. - 1 Nov. 2001, WHO Technical Report Series

[6] World Heart Federation, "Diagnosis and Management of Acute Rheumatic Fever Rheumatic Heart Disease", 2007 World Heart Federation, <http://www.world-heart-federation.org/> accessed 24 Feb 2015

[7] NHS Choice (Rheumatic Fever) http://www.nhs.uk/conditions/Rheumatic-fever/Pages/Introduction.aspx assessed 9 Feb. 2015

[8] "Rheumatic Fever and Rheumatic Heart Disease", Report of a WHO Expert Consultations, Geneva, 923.http://www.who.int/cardiovascular_diseases/resources/en/cvd_trs923.pdf, accessed 24 Feb 2015

[9] Sanjib Raj Pandey, Chiyaba Njovu, Choi-Hong Lai :"A Decision Support System for Diagnosis of Rheumatic Fever in Nepal", proceeding of 7th Asia Pacific Medical Informatics Conferences, Beijing, China, 22-25th October 2012

[10] Encyclopedia of Medical Decision Making, 2009, Contribution by J. Hilden, j.hilden@biostat.ku.dk

[11] Pandey Sanjib, Ma Jixin and Lai Choi-Hong (2014) "Development of Decision Support System for the Diagnosis of Arthritis Pain for Rheumatic Fever Patients: Based on the Fuzzy Approach" Journal of Algorithms and Computational Technology [in press for printing]

[12] Costas P. Pappis, ConstantinosI.Siettos "Chapter 15 Fuzzy Reasoning”, University of Piraeus, National Technical University of Athens School of Applied Mathematics and Physics, Athens, Greece.

[13] Allen, J.F., “Maintaining knowledge about temporal intervals”, CACM 26 (11) (1983) 832843.

[14] Ma Jixin and Brian Knight “A General Temporal Theory”, The Computer Journal 37(2), 1994, pp.114-123 